\section{Brain, Behavior and Evolution}

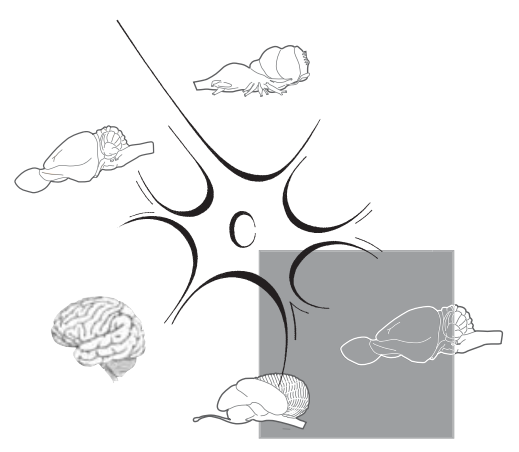

The past decade has witnessed a tremendous increase in the information available regarding gene expression in the brains of different species. This rapid accumulation of data has been fueled by advances in methodological approaches allowing for the efficient detection and measurement of mRNA, including techniques such as qRT-PCR, oligonucleotide and cDNA microarrays, RNA-seq, and different variants of serial analysis of gene expression (SAGE). Although these techniques provide a remarkable depth of information about transcriptional activity within a brain region, they are typically performed with homogenates of tissue, which dilutes sensitivity to cell type-specific effects. Thus, major challenges still remain at the intersection of the comparative analysis of brain structure and gene expression. Currently, one of the most serious obstacles is relating species differences in mRNA transcript levels to the vast complexity of diverse cell types distributed among layers of the neocortex and within subcortical nuclei. One strategy is to use laser capture microdissection to obtain samples for genome-wide expression profiling, as has been done for neocortical and dorsal lateral geniculate nucleus (dLGN) layers in rhesus macaques [e.g. Bernard et al., 2012]. However, laser cap-

\title{
Now that We've Got the Map, Where Are We Going? Moving from Gene Candidate Lists to Function in Studies of Brain Evolution
}

\author{
Chet C. Sherwood Tetyana Duka \\ Department of Anthropology, The George Washington University, Washington, D.C., USA
}

ture microdissection may be cumbersome for acquiring samples at a finer spatial resolution.

Two recent studies attempt to overcome these limitations by using in situ hybridization (ISH) to provide new insight into the role of cell type-specific regulation of gene expression in brain evolution [Mashiko et al., 2012; Zeng et al., 2012]. Such comparative gene expression atlases usher in a new era of brain mapping, yet they are firmly situated in the venerable old tradition of Brodmann, Vogt, Betz, von Economo, and others by providing detailed descriptions of phenotypic differences in brains across species. Today, however, the toolkit for observing neural phenotypes is much more sophisticated than the few histological stains that were available at the turn of the 20 th century. Modern brain mapping studies, which incorporate information from physiology, connectivity, cytoarchitecture, chemoarchitecture, receptor autoradiography, and gene expression, are essential guides for localizing hotspots of variation, pointing towards the features that make the brains of different species unique.

Mashiko et al. [2012] obtained ISH maps of the cerebral cortex, pulvinar, and dLGN from neonatal and fetal common marmosets, a small New World monkey, and compared them to data obtained from mice using 26 probes for transcription factors, cell adhesion molecules, zinc finger proteins, and other molecules with wellknown roles in neural development. The qualitative comparison between species revealed that genes previously found to regulate mouse cortical and thalamic development show broadly similar patterns of expression. However, when examined in detail, gene expression was conspicuously divergent between marmosets and mice in each of the structures investigated. The authors suggest that the specializations in marmosets relate to the evolution of visual acuity and higher cognitive functions characteristic of primates. In particular, a number of genes (Lhx9, Zic4, EphA7, Foxp2, and Cdh8) showed a gradient of expression in the dLGN of neonatal marmosets that was absent in mice. Additionally, in marmosets Btbd3 and CTGF were expressed exclusively in layer IV of the primary visual cortex, whereas these genes were more ubiquitous in their expression across the cerebral cortex of mice, with $C T G F$ being restricted to the subplate and layer V. Strikingly, these primary visual cortex-specific genes (Btbd 3 and CTGF) in marmosets were also expressed in area MT, a visual cortical region involved in motion detection that is unique to primates and has been proposed to serve as a

\section{KARGER}

Fax +4161306 1234 E-Mail karger@karger.ch www.karger.com
(C) 2012 S. Karger AG, Basel

0006-8977/12/0803-0167\$38.00/0

Accessible online at: www.karger.com/bbe
Chet C. Sherwood

Department of Anthropology, The George Washington University 2110 G Street, NW

Washington, DC 20052 (USA)

Tel. +1 202994 6346, E-Mail sherwood@ gwu.edu 
developmental organizing center for the growth of higher-order visual areas. Finally, the ISH maps of prefrontal cortex revealed a strong posterior expression gradient of EphA6 in marmosets, but not in mice, that might play a role in the development of increased cytoarchitectonic differentiation of heteromodal association cortex. Collectively, the results of this study suggest molecular mechanisms that may help to specify the differential parcellation of the thalamus and cerebral cortex in rodents and primates.

The study by Zeng et al. [2012] reported on ISH at a massive scale from 995 probes in two regions of adult human cortex, including the visual cortex (areas 17 and 18) and temporal association cortex (areas 20, 21 , and 22). The results were compared to those obtained previously in mice as part of the Allen Mouse Brain Atlas project. Differences in expression pattern (i.e. changes in intensity and/or laminar location) among cortical regions and species were scored on a qualitative scale. Of the genes investigated, $84 \%$ of the total showed consistency in expression pattern among the cortical areas investigated in humans. The striking cytoarchitectonic boundary between primary visual cortex (area 17) and extrastriate cortex (area 18) in humans was characterized by a modest $15 \%$ difference among the studied genes in their expression pattern, mostly due to higher or lower expression in layer IV compared to other layers. With respect to phylogenetic variation, $21 \%$ of the analyzed genes showed different expression patterns between humans and mice in the visual cortex. Most of these species differences were not among widespread cell types, but were rather localized to discrete cell populations in particular cortical layers. An especially remarkable finding was that more than half of the genes showing restricted expression in layer $\mathrm{V}$ of mice were diminished or absent in human layer $V$, yet there was a human-specific increase in expression of neurofilament, synaptic, and ion channel genes in large layer III neurons. The authors hypothesized that these patterns of gene regulation might reflect the enhancement of plasticity within supragranular cortical layers. Neurons in these layers participate in corticocortical association connectivity that is essential to higher order cognitive functions in primates.

These new studies are certain to stimulate further exploration of those genes that show the most divergent expression patterns among species. Now that these candidates have been identified, it is important to go a step further and investigate which isoform variants and posttranslational modifications of the related proteins might be significant for the way that the brain develops, performs computations, and undertakes physiological processes. This is especially critical since a substantial fraction of gene expression differences in the brain among species may conform to a neutral model of evolution, indicating that selection for function might not always be an important factor in driving apparent phylogenetic variation [Khaitovich et al., 2005]. Given the large scale of these recent mRNA mapping studies, it is not surprising that demonstration of a functional role for gene expression differences was beyond the scope of the research. However, the interpretation of such gene expression maps is not entirely straightforward because the link between biological function and differential enrichment for a particular gene in a specific cell type or cortical layer is complicated. This is because interacting networks of genes are complex and can be modified at many stages along the way to producing a protein. Take, for example, the expression of neuron cell adhesion molecule (NCAM) in the developing brain. There are three major isoforms of NCAM protein produced by alternative splicing of the mRNA transcript. Posttranslational attachment of polysialic acid (PSA) to one isoform of NCAM reduces its capacity for cell adhesion and therefore has effects on a wide range of dynamic cellular processes, such as cell migration, axonal growth and pathfinding, and synaptic plasticity. The different isoforms of NCAM, PSA-NCAM, and its cleavage fragments are each differentially up- and downregulated in the development of human prefrontal cortex along separate trajectories [Cox et al., 2009]. Therefore, knowledge of the mRNA transcript level of NCAM at single time point provides only a very partial picture of its function.

Moving from candidate gene lists to an understanding of how variation in expression reflects species-specific neurobiological function is also complicated because of the potential contribution of brain size. When comparing mammalian brains of such very different sizes (the difference in brain mass between humans and mice is $\sim 3,600$-fold; the difference in brain mass between marmosets and mice is $\sim 19$ fold), it is not clear which differences in gene expression levels are meaningful for cognition and which are simply a correlate of brain size to maintain functional equivalence as biophysical demands are scaled up. Some expected size-related modifications to gene expression are changes in metabolic pathways, cytoskeletal construction, signal transduction, and the diffusion distance of growth factors in developing brains. Many of these differences appear in comparisons of human neocortex to that of primates with smaller brains, presumably reflecting the energetic and structural requirements of neurons that have larger dendritic arrays and longer axons. Unfortunately, there are no similar comparative data on brain gene expression from other large brained mammals, such as elephants and cetaceans, to determine whether these are generalizable scaling effects. Consequently, unlike the allometric scaling rules that are known to govern species differences in the relative size of brain structures [Charvet et al., 2011], we have no similar rules to interpret phylogenetic variation in gene expression, and only a rudimentary basis for understanding scaling regularities in cell type distributions and neuronal morphology [Sherwood et al., 2010; Herculano-Houzel, 2012].

We anticipate that the future will hold intensified efforts to discover the function of species differences in gene expression patterns and levels, and to understand the networks of regulatory mechanisms leading to differential expression identified during this initial era of descriptive mapping studies. Historically, the acquisition of knowledge in neuroscience has proceeded from the descriptive to the functional. For example, interneuron types were initially identified only morphologically by early investigators using the Golgi impregnation technique; subsequently, electrophysiological experiments using intracellular recording methods revealed differences in spiking patterns and neuroanatomical studies demonstrated the specificity of synaptic terminations that characterize each interneuron type. In fact, exciting approaches already exist for characterizing the function of putative gene expression changes in brain evolution. Gene manipulation experiments are not feasible in humans, but studies in model organisms, such as the mouse and Drosophila melanogaster, can help reveal 
the molecular functions of newly evolved genes [Ranz and Parsch, 2012]. Also, by mapping regulatory elements for differentially expressed genes, interacting gene networks can be identified and examined for evidence of positive Darwinian selection. For example, in vitro studies of Foxp2, a gene that plays a role in the speech and language of humans, have shown that human-specific amino acid substitutions alter regulatory targets [Konopka et al., 2009]. Furthermore, transgenic mice expressing human Foxp2 exhibit differences in the anatomy and physiology of neurons in the striatum [Reimers-Kipping et al., 2010]. Another example is the study of SRGAP2, a recently evolved gene duplicate in humans. Experimental expression of human-specific SRGAP2 in mouse pyramidal neurons extends the phase of dendritic spine development and results in a higher density of spines [Charrier et al., 2012].

Finally, it is well known that protein diversity cannot be fully characterized by transcriptome analysis alone because of the significant influence of splice variation, allelic variation, RNA editing, microRNAs, and post-transcriptional regulation. Studies that have directly compared expression levels of mRNA (using either RNA-seq or microarrays) with protein in systems ranging from bacteria to human neocortex have reported only modest correlation coefficients, ranging from 0.2 to 0.5 [Fu et al., 2009]. Therefore, protein analyses are essential for understanding the functional significance of evolutionary changes in gene expression within specific cells and tissues. Knowing the subcellular localization of a protein provides an especially important clue about its potential function; hence, it is critical to examine species differences in protein expression using techniques such as fractionated preparations of tissue and immunohistochemistry in combination with high-resolution imaging modalities. Understanding the function of newly discovered candidate genes that might be responsible, in part, for anatomical or physiological differences among species is one of the key goals of comparative neuroscience. Attempts must be made to reduce the rapidly increasing gap between the vast amount of gene expression data that are becoming available and our knowledge of phylogenetic differences in neurobiological function. Generations of scientists will be working on these problems, now with useful maps of gene expression in the brains of different species to guide them.

\section{References}

- Bernard A, Lubbers LS, Tanis KQ, Luo R, Podtelezhnikov AA, Finney EM, McWhorter MME, Serikawa K, Lemon T, Morgan R, Copeland C, Smith K, Cullen V, Davis-Turak J, Lee C-K, Sunkin SM, Loboda AP, Levine DM, Stone DJ, Hawrylycz MJ, Roberts CJ, Joes AR, Geschwind DH, Lein ES (2012): Transcriptional architecture of the primate neocortex. Neuron 73:1083-1099.

-Charrier C, Joshi K, Coutinho-Budd J, Kim JE, Lambert N, de Marchena J, Jin WL, Vanderhaeghen P, Ghosh A, Sassa T, Polleux F (2012): Inhibition of SRGAP2 function by its human-specific paralogs induces neoteny during spine maturation. Cell 149:923-935.

- Charvet CJ, Striedter GF, Finlay BL (2011): Evodevo and brain scaling: candidate developmental mechanisms for variation and constancy in vertebrate brain evolution. Brain Behav Evol 78:248-257.

-Cox ET, Brennaman LH, Gable KL, Hamer RM, Glantz LA, LaMantia AS, Lieberman JA, Gilmore JH, Maness PF, Jarskog LF (2009): Developmental regulation of neural cell adhesion molecule in human prefrontal cortex. Neuroscience 162:96-105.
Fu X, Fu N, Guo S, Yan Z, Xu Y, Hu H, Menzel C, Chen W, Li Y, Zeng R, Khaitovich P (2009): Estimating accuracy of RNA-Seq and microarrays with proteomics. BMC Genomics 10: 161.

Herculano-Houzel S (2012): Neuronal scaling rules for primate brains: the primate advantage. Prog Brain Res 195:325-340.

Khaitovich P, Pääbo S, Weiss G (2005): Toward a neutral evolutionary model of gene expression. Genetics 170:929-939.

Konopka G, Bomar JM, Winden K, Coppola G, Jonsson ZO, Gao F, Peng S, Preuss TM, Wohlschlegel JA, Geschwind DH (2009): Human-specific transcriptional regulation of CNS development genes by FOXP2. Nature 462:213-217.

Mashiko H, Yoshida AC, Kikuchi SS, Niimi K, Takahashi E, Aruga J, Okano H, Shimogori $\mathrm{T}$ (2012): Comparative anatomy of marmoset and mouse cortex from genomic expression. J Neurosci 32:5039-5053.
Ranz JM, Parsch J (2012): Newly evolved genes: Moving from comparative genomics to functional studies in model systems: how important is genetic novelty for species adaptation and diversification? Bioessays 34:477-483.

-Reimers-Kipping S, Hevers W, Pääbo S, Enard W (2010): Humanized Foxp2 specifically affects cortico-basal ganglia circuits. Neuroscience 175:75-84.

- Sherwood CC, Raghanti MA, Stimpson CD, Spocter MA, Uddin M, Boddy AM, Wildman DE, Bonar CJ, Lewandowski AH, Phillips KA, Erwin JM, Hof PR (2010): Inhibitory interneurons of the human prefrontal cortex display conserved evolution of the phenotype and related genes. Proc R Soc B 277: 1011-1020.

Zeng H, Shen EH, Hohmann JG, Oh SW, Bernard A, Royall JJ, Glattfelder KJ, Sunkin SM, Morris JA, Guillozet-Bongaarts AL, Smith KA, Ebbert AJ, Swanson B, Kuan L, Page DT, Overly CC, Lein ES, Hawrylycz MJ, Hof PR, Hyde TM, Kleinman JE, Jones AR (2012): Large-scale cellular-resolution gene profiling in human neocortex reveals species-specific molecular signatures. Cell 149:483-496. 